\title{
Managing Adaptive Information Projects in the Context of a Software Developer Organizational Structure
}

\author{
Pawel Cichon ${ }^{1}$, Zbigniew Huzar ${ }^{1}$, Zygmunt Mazur ${ }^{1}$, Adam \\ Mrozowski ${ }^{1}$ \\ 1Wroclaw Institute of Technology, Wybrzeze Wyspiańskiego 27, 50-370 Wroclaw \\ \{Adam.Mrozowski, Pawel.Cichon, Zbigniew.Huzar, \\ zygmunt.Mazur\} apwr.wroc.pl
}

\begin{abstract}
The article describes an unique production process and an organizational structure for adaptive software development project execution with the use of SPEM profile of UML language. The organization of a company presented in this paper is based on neutral matrix model including elements essential for remote execution of adaptive projects. The presented solution enables to react more quickly to changes during execution, minimize the risk of incorrect functional requirement specification and improve the final product quality through strong verification of every phase of the productive process.
\end{abstract}

\section{Notation}

The presentation of the research described in this article is based on the Software Process Engineering Metamodel (SPEM), which is used to describe a concrete software development process or a family of related software development processes. An object-oriented approach has been chosen to model a family of related software processes and the UML profile has been used as a notation. This introduction covers only a minimal set of process modeling elements necessary to describe the presented processes. More details can be found in SPEM specification delivered by OMG [OMG 2005]. At the core of SPEM is an idea that a software development process is a collaboration of abstract active entities called process roles that perform operations called activities on concrete, tangible entities called work products. The overall goal of the process is to bring a set of work products to a well-defined state. The fragment of SPEM conceptual model is presented in the Figure 1. 


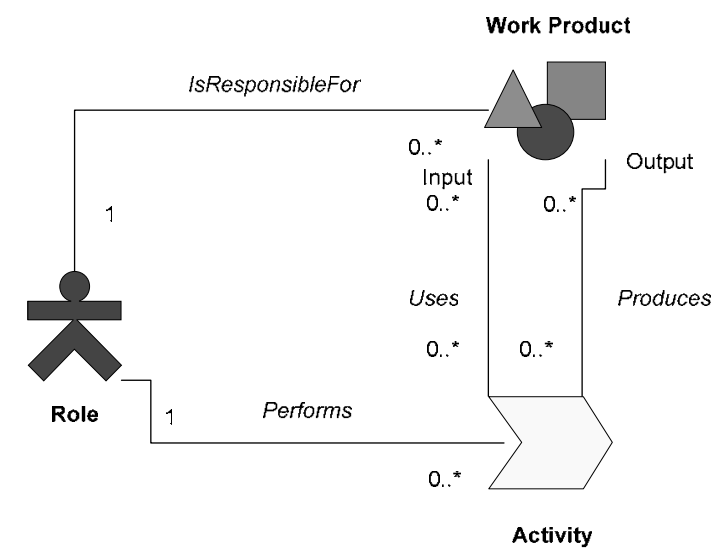

Figure 1. The SPEM Conceptual Model: Roles, Work Products, and Activities.

The notation used in the article for the presented conceptual model includes the following elements:

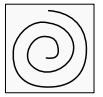

This symbol indicates a stand alone, complete, end-to-end process class - Process. It is distinguished from normal ProcessComponents ${ }^{1}$ by the fact that it is not intended to be composed with other components. The class Process can also represent a family of processes, which is a process component out of which multiple overlapping processes can be defined.

ProcessRole is a subclass of ProcessPerformer described below.

ProcessPerformer defines a performer for a set of WorkDefinitions in a process. ProcessPerformer has a subclass ProcessRole. ProcessPerformer represents the "whole process" or one of its components in an abstraction, and is used to own WorkDefinitions that do not have a more specified owner.

Phase is a specialized subclass of WorkDefinition thus its prerequisite defines the phase entry criteria and its goal (often

1 ProcessComponent constitutes a chunk of process descriptions that are internally consistent and may be re-used with other ProcessComponents to assemble a complete process. 
called a "milestone") defines the phase exit criteria. Phases are defined with the additional constraint of sequentially; that is, their enactments are executed with a series of milestone dates spread over time and often assume minimal (or no) overlap of their activities in time.

WorkDefinition is a class that describes the work performed in the process. Its main subclass is Activity, but Phase is also a subclass of WorkDefinition.

Activity is the main subclass of WorkDefinition. It describes a piece of work performed by one ProcessRole: the tasks, operations, and actions that are performed by a role or with which the role may assist.

WorkProduct is anything produced, consumed, or modified by a process. It may be a piece of information, a document, a model, source code, and so on. WorkProduct describes one class of work product produced in a process.

Document is a category of WorkProduct.

UMLModel is a category of WorkProduct.

\section{Introduction}

The complex information projects are difficult to estimate because of multiresource fullness and a short life cycle, which indicates a short period between next modifications or extensible development of application functionalities [Boehm 1981]. Therefore complex systems are often adapted to budget during process of execution, even though in a canonical model of the project management a business contract is entered into, on the base of a cost of subsystems and tasks, the project is decomposed. Most important is to create an information system that makes business run as efficient as a client wants, and fit in the time frames and the client's budget. Contract is signed mostly on the base of non-functional requirements with most important elements that expose main 
priorities: scope ${ }^{2}$, cost and time. This type of contract is called adaptive contract ${ }^{3}$, and information projects thus based are called adaptive projects. These projects, as other information projects types ${ }^{4}$ such as waterfall, agile, optional scope etc., are successful, when they meet functional and non-functional requirements, which determine the quality of the final product. The difference is that adaptive projects emphasize extensively non-functional requirements. The non-functional requirements constitute the basis for functional specification, which is postponed to further phases of the adaptive project (Figure 2, Figure 3, Figure 5).

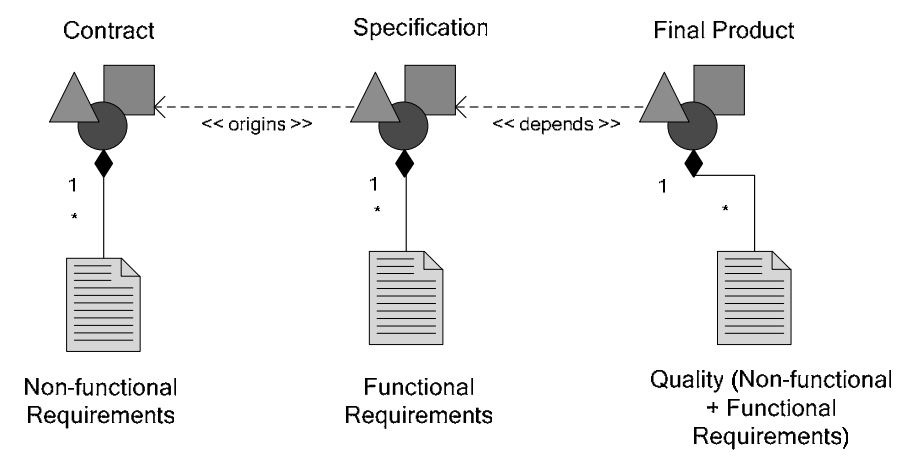

Figure 2. Requirements in the context of adaptive project life cycle

Specification of functional requirements during project execution poses the main difficulty for software developer because continuous changes of requirements cause that it is difficult to foresee entire project realization cycle at the beginning. Therefore a software developer has to respond as quickly as possible to changes at the project execution and functional requirements must meet the actual client's needs with reference to production capabilities, cost and time limits.

\footnotetext{
${ }^{2}$ Scope defines specification of business processes that should be covered by the entire project.

${ }^{3}$ Terms adaptive contracts and adaptive projects used by the authors, are also called fixed price contracts and fixed price projects for the sake of stable nonfunctional requirements (the price set by the client covers resource function, project complexity and its time duration). Adaptive projects are fixed price projects, which allow specifying functional requirements during entire project life cycle (adapting functional to non-functional requirements), this is the reason for "adaptive" adjective usage. Adaptive contracts are fixed price contracts in view of adaptive projects (term adaptive contract is used mainly for syntactic coherence with adaptive project term).

${ }^{4}$ Projects are named mainly for the sake of the most important characteristic, which is the basis for non-formal project typization.
} 
The article presents description of adaptive type of an information project, unique execution process of an adaptive project and company organizational structure for remote adaptive project execution with the use of SPEM language [OMG 2005, Sas 2003]. Originality of the described approach relies in parallelism of implementation and design of productive process sub-phases (Figure 5) in the context of evolving functional requirements and in setting consultants and project data set in a standard matrix organization structure of an information company. The presented solution allows quicker reaction to the functional changes that may appear during the development process.

\section{Adaptive process and project realization method}

The traditional approach to an information project execution presupposes that the phases of design, implementation, testing and migration occur sequentially. The next phase starts upon completion of the preceding phase ends, therefore the first versions of the working system are delivered to the client relatively late because in the last phases of the project life cycle. Adaptive process is guided by a different approach to the traditional one mainly because it delivers the working application to the client as soon as possible, through making design sub-phase parallel to implementation sub-phase, making them iterative and taking advantage of phase interdependency (Figure 2, Figure 4). This leads to the creation of a sub-process oriented to the delivery of the project development results to the client in the form of functional subsystems.

An adaptive project allows for functional requirement extension during the project execution. When an adaptive contract is signed, mainly its range is specified, in terms of business processes that will be covered by the entire project. There was proved that a realization of just 20 percent of the functionality makes 80 percent of client's satisfaction [Popendieck 2004], it means quality and business value of the final product. An adaptive project promotes this point of view first of all by emphasizing quick delivery of the most important 20 percent of functionality and the project guidance by the customer. The client verifies and specifies functionality of the system and in such a way affects the whole informational system execution process. Continuous process adaptation to the customer requirements to obtain the highest possible business value of the final product describes the adaptive process, which is fundamental for adaptive projects.

The analysis of project management methods (RUP, USDP) leads to conclusion that most of them have no strict marginal conditions ${ }^{5}$. Such an

\footnotetext{
${ }^{5}$ Environment parameters that must be met for safe application of the methodology.
} 
instance brings about solutions that are oriented to predictability ${ }^{6}$ in unpredictable processes which increase the project failure risk. A method dedicated to the case of an adaptive project, preceded by signing a fixed price type contract is presented below. The adaptive projects are partially unpredictable, because of defining functional requirements during process realization and that couldn't signify unpredictable and uncontrolled process (called realization chaos). Therefore a repetitive realization process and right organizational structure has to be created that assure control over unpredictability and let react efficiently to changes of functional requirements and processes while realization of the project. Reaction to functional requirements changes needs tight collaboration between the team members and domain experts (consultants) [McConnell 1998]. This collaboration goes beyond standard business roles (every worker should be regarded as an expert and consultations should constitute a preferred solution of problems) and preparation of an evolving process to meet continuous functional requirement realization needs regular audits (evaluation of milestone progress).

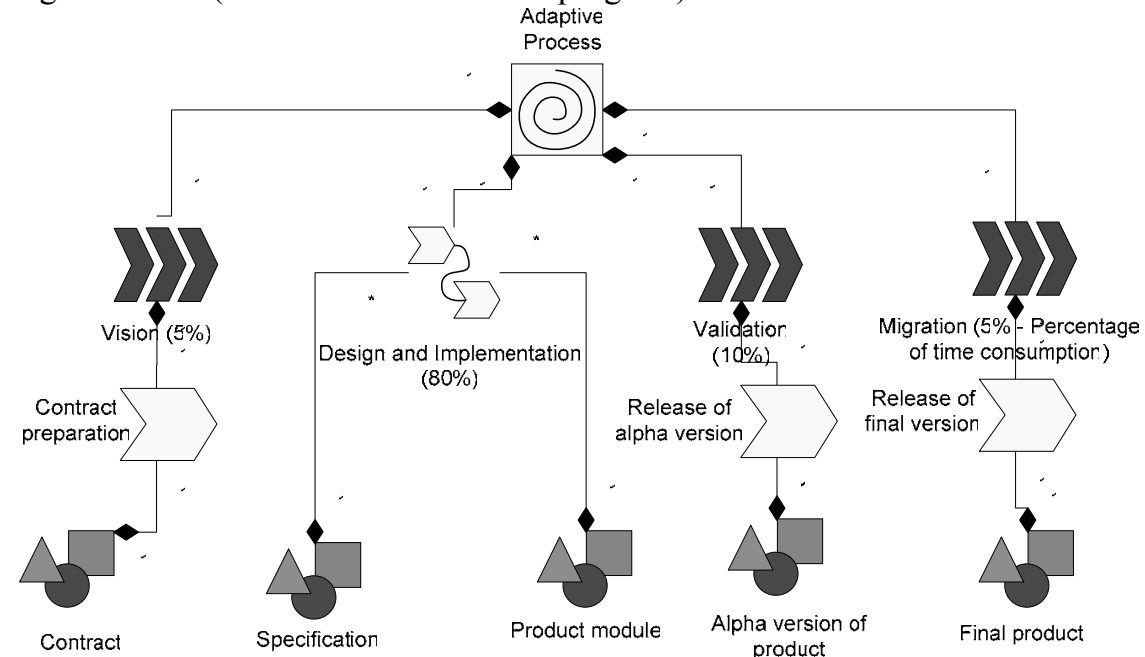

Figure 3. The execution process of adaptive projects with marked phases and artifacts.

The project execution process starts with the vision phase. In that stage consultants and customer representatives focuses, among other things, on defining of a document describing non-functional requirements that are a part of the contract (Figure 3). The consultants, working together with domain experts, create a list of business processes that have to be covered by a system. It is

${ }^{6}$ Predictable solutions are the solutions that have all functional and non-functional requirements set before the project starts. This allows for creating of an entire project at the very beginn. 
important to describe points of integration of the new system, existing infrastructure and external processes. The goal of the vision phase is to define the project scope in the context of non-functional requirements and point out the most important threats. Furthermore, there is a coordinator and domain experts (on the customer's side) selected, responsible for functional requirements of the system specification. They decide about supplementation or removal of a specified functionality proposed by the consultants. After contract signing the project team commences work.

The method presented here assumes an iterative approach as regards the risk ${ }^{7}$ in the design and implementation phases. Extensive and narrow ${ }^{8}$ implementation $^{9}$ of many components ${ }^{10}$ [Kasprzyk 2004] parallely comes true. The objective is a quick stabilization which entails partial implementation of base software and hardware components, associated with functionality that has high business priorities and definition of interfaces, the cooperation rules and environment. From the customer's viewpoint the approach above has a psychological value because early implementation of key functionalities helps to stabilize the high level of customer's involvement in the project and that facilitates smooth project execution [Cockburn 2003]. It is very important because in iterative designing directed to the client, customer decides about elements introduced in successive iterations, demanding implementation of the most important modules from his point of view. A single iteration ${ }^{11}$ consists of sub phases, presented on Figure 4.

The most important activities in specified sub phases are as follows:

- Initiation. The team members create a list of use cases and their extensions. Moreover, all the possible risks should be identified. The sub-phases' goal is to specify the functional elements of a system that should be implemented in a coming iteration.

- Planning. Choice of the functionalities (their verification) that must be implemented in the context of a business value for a client. Setting the iteration scope by removing less important functional requirements from the planning phase.

- Implementation. Putting into practice those functionalities which are consistent with the verified requirements list. The sub-phase ends with a release of the tested internal ${ }^{12}$ version of a sub system.

${ }^{7}$ For the early iterations the most risky elements are chosen.

${ }^{8}$ Implementation that contains all the most important elements of the system from the customer and supplier's perspective.

${ }^{9}$ Programming activities that include only frames of functionalities. These frames may be extended in further iterations.

${ }^{10}$ Subsystem that contains one or several coherent use cases.

${ }^{11}$ Set of activities that lead to creation of a functional system module.

12 Internal testing means verification of partially finished product and is made during production process (Verification sub-phase of design and implementation phase). 
- Verification. Representatives of the developer and the client organize a meeting to discuss the implemented functionality. They point to weakness and strengths parts of a solution, identify potential problems and indicate possible extensions. The goal is to show the best possible way to develop the existing application.

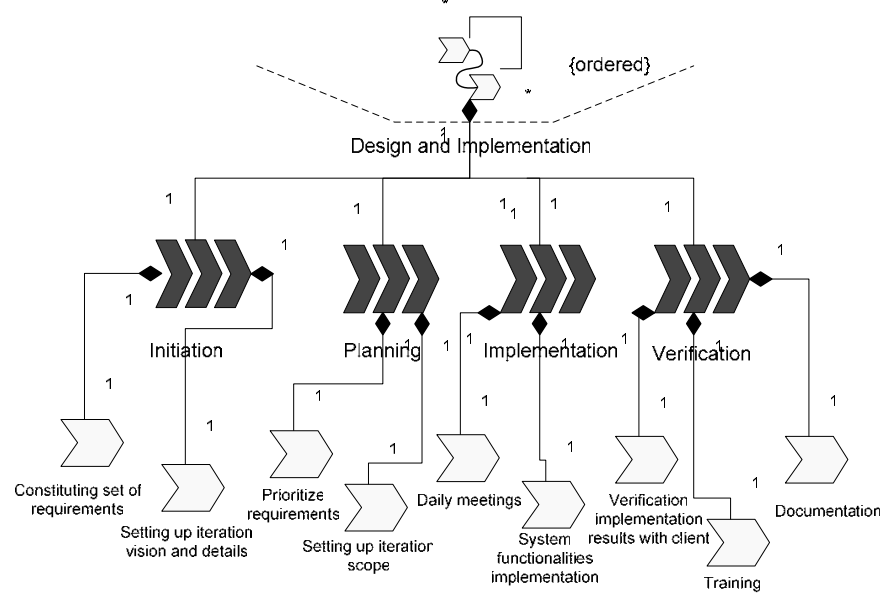

Figure 4. Decomposition of a design and implementation phase into sub phases and activities.

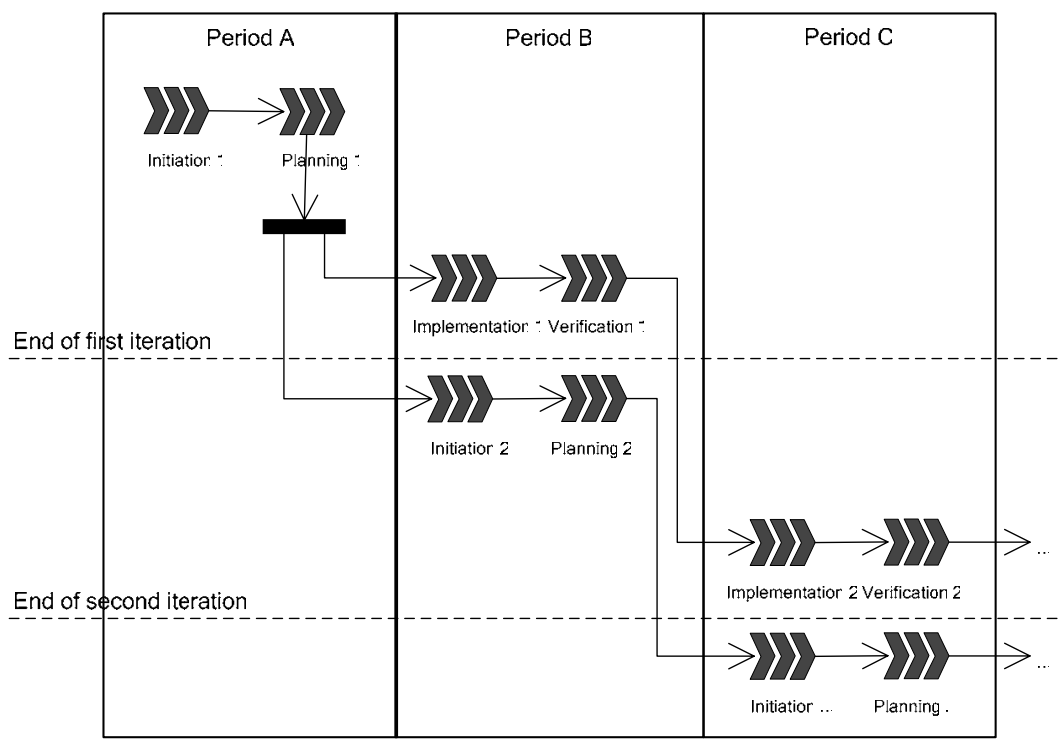

Figure 5. Parallelism of iterations of design and implementation sub-phases. 
The last iteration in the design and implementation phase ends with the release of the final, tested alpha version of the system. After this project moves to the validation phase. The customer can now test whole system and report errors. Requests for supplementation or removal of any functionality cannot be reported on this level. If a necessary requests change occurs, they may be written as an extra-paid task. After the external ${ }^{13}$ testing has been completed and the errors corrected the system is transferred from a test environment to production servers. This stage is called the migration. As the result of analyze of the projects that had not been completed successfully [Jonson 2001] the presented process contains the following key points:

- Self-organizing teams that cannot exceed 10 persons. During the iteration project the managers and the team leaders do not plan tasks for the team members. The teams are self-organized. Members share the tasks among themselves and decide about time frames necessary for each task execution. Only the disputes are solved by team leaders. Strong task orientation is preferred and the whole team should responsible for meeting the deadline [Highsmith 2002].

- Project executed with the use of iterations (Figure 5). Additionally, for projects realized by many distributed teams it's suggested to do two first iterations in a centralized structure at one site (Figure 6). A leaders' team should be created for these iterations. A common team should contain one or two most experienced programmers from the future remote teams. During these two iterations the greatest emphasis is put on requirement specification, which includes the most important components and their interfaces. The components related with the most important for the customer business runs are created more efficiently due to direct communication and cooperation of the best programmers. When the basis are ready, the main programmers are coming back to their teams and acting as team leaders. Further work can be done remotely. This way every team has it's leader but also a person who has created fundaments of a system. Moreover, thanks to the team leaders' direct cooperation in the first two iterations further communication between teams has also improved during the whole project.

- Adaptive planning guided by the customer. The client takes a part in building functional requirements after each iteration and may make decisions in cooperation with developers' consultants. Customer has direct influence to order of use case implementation and may choose these of the highest business value.

\footnotetext{
${ }^{13}$ External testing is synonym to validation and means tests performed by customer after final product release by software developer.
} 


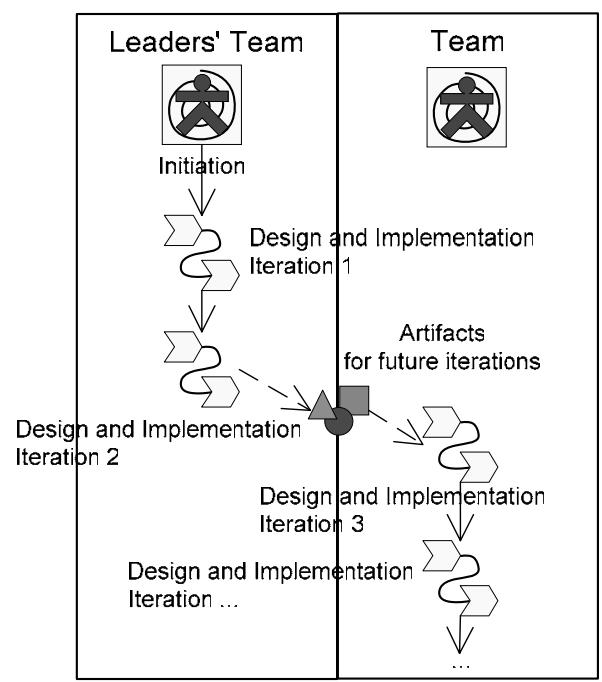

Figure 6. Iterative implementation based on distributed teams.

- Adding a new functionality to an on going iteration has to be locked. Consultants, team members or the customer's representatives are not allowed to add new functionalities to the running iteration. Team leaders together with the project managers are acting as a firewall that isolates the team members from customer's change requests. If changes appear, the project managers negotiates their implementation in the future iterations (putting into tasks queue) [MacCormack 2001].

- Daily meetings of the entire team. Decisions are made in maximum one hour.. This method promotes the approach that wrong decisions are better than no decisions. Wrong decision can be corrected in the future iterations but no decision leads to the project stagnation [MacCormack 2001]. The tasks set for one day must be completed during that day. If any problems appear their are discussed at a next day meeting.

- An internal version release at the end of each iteration and a demo version for the client at least every two iterations.

\section{Software developer organizational structure as regards of adaptive projects execution.}

The organizational structure presents a network of organization bonds ${ }^{14}$ and dependencies, which constitute a connection among all company resources, both

\footnotetext{
${ }^{14}$ Information exchange channel.
} 
human as well as material. Moreover, it describes distribution of work activities and employees' hierarchy, shows correlation between functions and activities and specifies a responsibility allocation. The following bonds occur in organization [Cichon 2004]:

- Technical (task oriented) - these bonds connect positions that are involved in task execution (implementation). Following stereotypes shown in Figure 7 $<<$ produces $>>,<<$ elaborates $>>$.

- Functional (function oriented) - these bonds connect positions that are involved in decision management and tasks planning. Following stereotypes shown in Figure $7<<$ supervises $>>,<<$ is subordinated $>>,<<$ consults $>>$, $<<$ cooperates $>>$.

The method of adaptive fixed price project execution presented in the previous chapter realization is efficient only when it is based on an appropriate organizational model. Organizational models the best for the presented process are task-oriented structures like the matrix model [Cichon 2004]. The matrix model, depends on managers' and team leaders' competences, can be divided to: weak - when a manager allows the team leader to allocate tasks to the team members, strong - when the manager assigns the tasks and neutral - when the manager together with team members perform task allocation. Whatever the model the manager's role should always be focused on planning, scheduling and budgeting and the team leaders' role on technical supervision. Figure 7 presents an organizational model drafted for remote run of process presented in the previous chapters and is based on neutral matrix model.

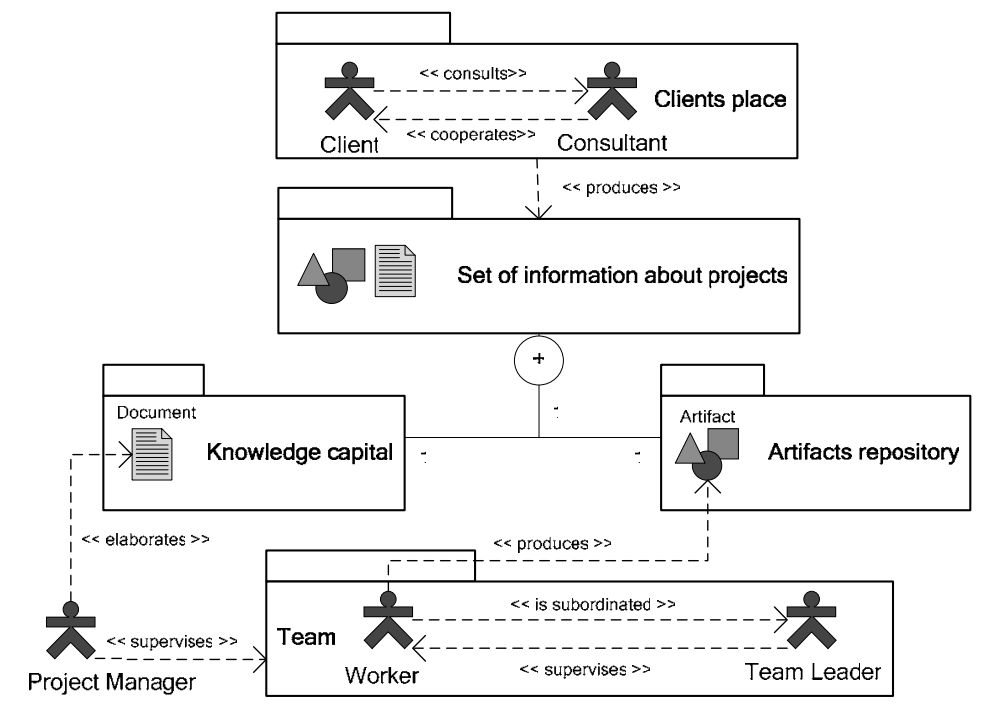

Figure 7. A neutral matrix model for presented process of fixed price projects execution. 
A team member is directly subordinated to the team leader, who verifies the work progress and is responsible for implementation quality. Moreover, leaders are responsible for implementation coherency, repository data update and team members' training. Teams should be grouped by specialization. In this way the team leaders and project managers can be certain that their teams have the necessary knowledge to carry out the assigned tasks. Project managers are responsible for scheduling, budget verification, changes and risk management. They may also with cooperation of team leaders allocate tasks packages to teams. The executed assignment is based on functional requirements and system decomposition delivered by the consultants working at customers' site, who are responsible for the system analysis and decomposition into modules ${ }^{15}$. Project managers, consultants and team leaders are obliged to report work progress at weekly project meetings.

The set of information about projects includes knowledge capital and remote repository, which is extremely important for remote cooperating teams and consultants. Firstly, it allows keeping project big picture ${ }^{16}$; secondly, it's essential for future projects and maintenance of already-designed systems. Thirdly, it's an integral part of intellectual capital of a organization. It contains the knowledge about previous projects that could be used for result comparison, drawing conclusions and risk minimization. The knowledge capital covers project documentation - schedule, budget, cost tables, risk calculations, teams and resources lists and other data important from the managing perspective. The remote repository is a set of artifacts - product source codes with descriptions, managed by a versioning system with changes annotations. Every worker must have uninterrupted access to the set of information about the projects, as presented in Figure 7. This is the only way in which the workers may understand their role in the project while working remotely and localize assigned tasks in whole project perspective.

\section{Summary}

The article is the cooperation result of IMG consultants with scientists from Wroclaw University of Technology. The developer as well as the customer take the risk of adaptive contracts execution which makes the specialized method of leading unpredictable projects is necessary. This method presupposes the consideration of characteristic elements of adaptive projects such as:

- Fixed price (adaptive) contract,

- Process controlled by the customer,

- Parallelism of design and implementation sub-phases (Figure 5),

- Specification of functional requirements in the course of project execution,

${ }^{15}$ A functional part of the system.

${ }^{16}$ Big picture means complete information data set, which describes the project execution. 
- Delivery to the customer an early versions of subsystems during the project development,

- Absence of long-term planning ${ }^{17}$.

There aren't any management methods dedicated for adaptive projects and the application of universal solutions to adaptive production processes leads to project failure or calling project into question in 70\% [Popendieck 2004]. Due to proposed in the paper adaptive process and its orientation to changes of functional requirements the customer may control development process more efficiently and the software developer may react more quickly to the customer's requirement changes. The presented solution proposes a modern organizational structure for every project execution, which ensures stable and clear communication between the teams and the customer, essential for quick response to changes. Thanks to it and directly to consultants working on the customers' site, the team members have continuous access to the functional requirements and domain experts. Moreover, the presented structure is opened for telecommuting [Cichon 2004] and actually put into practice by Swiss consulting group IMG. The first fixed price projects with the use of described method were completed successfully, what constitutes great potential of project execution in the future.

\section{References}

[Boehm 1981] Boehm, B., Software Engineering Economics, Prentice-Hall, 1981

[Cichon 2004] Cichon, P., Huzar, Z., Remote project management using modern organizational forms, ISAT Proceedings, Wroclaw University of Technology Publishing House, 2004, pp.39-47.

[Dubielewicz 2003] Dubielewicz, I., Sas, J., SPEM/UML Profile in business processes of project management, WNT, 2003, pp. 479-495.

[Cockburn 2003] Cockburn, Alistair., Characterizing People as Non-Linear, FirstOrder Components in Software Development, http://alistair.cockburn.us/ crystal/articles/cpanfocisd/ characterizingpeopleasnonlinear.html, 2003.

[Highsmith 2002] Highsmith, J., Agile Project Management: Principles and Tools, Cutter Consortium Executive Report, 2002.

[Jonson 2001] Jonson, J., Standish Group Chaos Report, 2001.

[Kasprzyk 2003] Kasprzyk, A., SelectPerspective ${ }^{\mathrm{TM}}$ Methodology, Components in practice, WNT, 2003, pp.121-126.

[MacCormack 2001] MacCormack, A., Product-Development Practices That Work, MIT Sloan Management Review, 2001.

[MacCormack 2001] MacCormack, A., Verganti, R., and Iansiti, M., Developing Products on Internet Time: The Anatomy of a Flexible Development Process, Management Science, 2001.

\footnotetext{
${ }^{17}$ Short term planning concentrates only on planning coming iteration instead of planning
} of whole project. 
[McConnell 1998] McConnell, S.: Software Project Survival Guide, Microsoft Press, 1998.

[OMG 2005] OMG, Software Process Engineering Metamodel Specification, OMG Press, 2005.

[Poppendieck 2004] Poppendieck, M., Website: www.poppendieck.com, 2004. 